\title{
Can the use of radiography of arms and legs in accident and emergency units be made more efficient?
}

\author{
M C CHARNY, W P ENNIS, C J ROBERTS, $\mathrm{K} T$ EVANS
}

\begin{abstract}
The efficiency of the selection of patients with injured arms and legs for radiography was investigated. The analysis was based on data on presenting signs and symptoms collected in a multicentre study organised by the Royal College of Radiologists working party on the effective use of diagnostic radiology. The work was carried out in eight accident and emergency units in England and Wales. With the help of various computer simulation techniques a combination of signs and symptoms that might usefully improve present practice was sought. The results suggest that for injuries of arms and legs the clinical determinants of bony injury cannot be refined further to improve current selection for radiography.

This study shows that existing clinical practice is probably as good as it can be.
\end{abstract}

\section{Introduction}

Some radiologists believe that radiography of arms and legs is performed too often among patients attending accident and emergency departments with acute musculoskeletal injuries. ${ }^{1-3}$

\footnotetext{
Departments of Epidemiology and Community Medicine and Diagnostic Radiology, University of Wales College of Medicine, Heath Park, Cardiff CF4 4XN

M C CHARNY, MB, MFCM, lecturer in epidemiology and community medicine W P ENNIS, PHD, research officer

C J ROBERTS, FFCM, FRCR, professor of epidemiology and community medicine K T EVANS, FRCP, FRCR, professor of radiology

Correspondence to: Dr Charny.
}

They suggest that the doctors requesting such radiography are often inexperienced and may refer too many patients for radiography because of heavy workloads, fears of medical litigation, patients' expectations, and the lack of explicit agreed criteria for selecting patients for $x$ ray investigation.

In Britain, as elsewhere, there has been a steady increase in the number of investigations requested. The number of radiographer units of work in England has risen at an annual rate of $5 \%$ since $1966,{ }^{4}$ implying a doubling of workload every 14 years. About 3.5 million radiographs of arms and legs are taken in Britain each year at a cost to the National Health Service of about $135 \mathrm{~m} .{ }^{5}$ There is now considerable interest in methods to limit this expansion, which some believe does not always improve outcome..$^{6-10}$ The Royal College of Radiologists working party on the effective use of diagnostic radiology has already investigated the use of radiography of the skull in head injuries, ${ }^{11-13}$ preoperative radiography of the chest in elective non-cardiopulmonary surgery, ${ }^{14}$ and abdominal radiography in the management of patients with acute abdominal symptoms. ${ }^{15}$

The working party recently undertook a multicentre study of the use of radiography of the arms and legs in injured patients attending eight accident and emergency departments in England and Wales. ${ }^{16}$ The working party stated that "guidelines for selection of patients for extremity radiography would reduce variability in its use and improve the quality of present practice." ${ }^{16} \mathrm{We}$ describe attempts to develop such guidelines using three types of algorithm, based on history and examination, to reduce the number of radiographs taken by identifying, before investigation, which patients have sustained a fracture and which have not. This approach offers several potential benefits: fewer patients would be exposed to the hazard of radiation and the discomfort of a test for no clinical gain; resources could be diverted from unnecessary radiography and put to more worthwhile uses; and doctors might benefit as guidelines offer a readily available standard by which courts can measure acceptable practice. ${ }^{17}$ 


\section{Methods of data collection}

The study methods have been described previously. ${ }^{16}$ Of 9105 patients with injured arms and legs who were referred for radiography, 4980 had at least one radiograph of the arm taken and 4125 had at least one taken of the leg. In the 9105 patients referred for radiography 5098 radiographs of the arm and 4438 of the leg were reported. The doctor in the accident and emergency department recorded the presence or absence of 10 signs and symptoms, how long ago the injury had occurred, and any previous attendances at an accident and emergency department for the same injury for each site for which a radiograph was requested. This information was entered on a wallet specially designed for the study. The 10 symptoms and signs recorded were: severe signs (bony deformity or crepitation), pain at rest, pain on weight bearing or movement, generalised tenderness, bony point tenderness, limited movement, swelling, bruising, abnormal distal vascular or tendon function, and impaired sensation. If and when the $x$ ray films became available for reporting the findings were recorded with an eight point classification: greenstick fracture, simple fracture, complicated fracture, other bone or joint disease, dislocation of joint, soft tissue swelling, foreign body in soft tissue, and normal. The performance of the best guidelines was similar for radiographs of arms and legs. Here we discuss the results of radiographs of arms alone.

Of the 4980 patients with injured arms who had had at least one radiograph taken, $4659(94 \%)$ were included in the analysis. Of the 321 patients who were excluded, 147 had attended previously for the same injury or were uncertain whether they had attended previously, 158 did not have results of radiography available, and 16 had no recall of the time between injury and attendance. Only one radiograph per patient was included in the analysis.

For the purposes of the analysis a fracture was defined as a greenstick fracture, a simple fracture, a complicated fracture, or dislocation of the joint. A non-fracture was defined as a normal result, a foreign body in soft tissue, a swelling of soft tissue, or other bone or joint disease. This division by the presence or absence of a fracture was dictated by the uses envisaged for any algorithm that might result. Of the 4659 patients in the analysis, 1667 (36\%) had fractures and $2997(64 \%)$ did not.

The variables used in the analysis were age (up to $16,16-59$, and over 59 years), time since injury (less or more than 12 hours), sex, and the symptoms and signs listed above.

\section{Development of guidelines}

With the use of two approaches several algorithms were identified and their effects simulated. One approach used discriminant analysis, a multivariate statistical technique in which various combinations of variables are used to distinguish as accurately as possible between two or more categories of cases. The variables discriminate between groups of cases and predict into which category each falls, based on the values of these variables. The discriminant analyses were performed using the statistical package for the social sciences. ${ }^{18} 19$ Prior probabilities were assigned according to the size of the populations defined as having fractures and non-fractures $(0.36$ and 0.64 , respectively).

The second approach may be called Boolean because it uses the and/or logic of Boolean algebra. Two different methods of choosing the criteria for guidelines were used. With one.an odds ratio calculation was applied to the 10 symptoms and signs recorded, with (guideline I) or without (guideline III) the addition of the remaining variables-namely, age, sex, and time since injury. For example, guideline III is based on referring for radiography those with at least one of the following: severe signs, swelling, or limited movement. The odds ratio is the ratio of the odds in favour of the sign or symptom being present in patients defined as having fractures to the odds in favour of the same sign or symptom being present in those defined as having non-fractures. These two groups of variables allowed protocols to be developed for symptoms and signs alone as well as for all collected variables. The variable with the highest odds ratio was identified, and all patients who had this variable were removed from the analysis. ${ }^{20}$ The same process was repeated on the remaining records, and those records positive for the most significant variable were again removed. ${ }^{21}$ At each stage, therefore, the variable that best distinguished those with fractures and those without was chosen. The process was repeated until no significant difference was found $\left(\chi^{2}\right.$ test, $p>0.05$ ). The second method was also applied to the 10 symptoms and signs, with (guideline II) or without (guideline IV) the addition of the remaining variables, but at each stage the variable that correctly identified the higher proportion of fractures (whether or not it distinguished well between patients with fractures and those with non-fractures) was chosen as a criterion for radiography. Again, this process was repeated until no significant difference was found $\left(\chi^{2}\right.$ test, $p>0.05$ ).

The following measures were used to assess the performance of the protocols obtained: the proportion of those with fractures that would have been identified correctly (sensitivity), the proportion of those without fractures who would have been identified correctly (specificity), and the 3 proportion of the population who would have had $x$ ray films taken if the $\stackrel{\mathbb{Q}}{\mathbb{Q}}$ protocol had been applied.

\section{Results}

Below we discuss the results obtained using five different ways of combining symptoms and signs that best discriminated between fractures. and non-fractures. They are referred to as A, B, C, D, and E. When applied $\overrightarrow{\vec{F}}$ to a patient with known symptoms and signs the combination resulting from $\stackrel{\rho}{\rightarrow}$ a discriminant analysis produced two scores, one for the presence of a? fracture and one for the absence of a fracture. For example, function $C \underline{\underline{B}}$ assigned scores for fractures or non-fractures based on severe signs, pain at $\frac{\bar{\sigma}}{\overline{2}}$ rest, bony point tenderness, limited movement, and swelling. The patient $\Phi$ was classified as having a clinical fracture when the fracture score was larger than the non-fracture score, and as having no fracture when the opposite was $₫$ true.

TABLE I-Performance of discriminant functions. Values are numbers $(\%)$ of patients

\begin{tabular}{lccc}
\hline & & & $\begin{array}{c}\text { Proportion of } \\
\text { population } \\
\text { who would } \\
\text { have had } \\
\text { radiographs } \\
\text { taken }\end{array}$ \\
$\begin{array}{l}\text { Discriminant } \\
\text { function }\end{array}$ & Sensitivity & Specificity & $\begin{array}{c}\text { (a) } \\
\text { A }\end{array}$ \\
B & $550(33)$ & $2761(92)$ & $786(17)$ \\
C & $485(29)$ & $2768(92)$ & $756(16)$ \\
D & $480(29)$ & $2807(94)$ & $675(15)$ \\
E & $481(29)$ & $2805(94)$ & $669(14)$ \\
\hline
\end{tabular}

TABLE II-Performance of Boolean algorithms. Values are numbers $(\%)$ of patients

\begin{tabular}{lrrc}
\hline & & $\begin{array}{c}\text { Proportion of } \\
\text { population } \\
\text { who would } \\
\text { have had } \\
\text { radiographs } \\
\text { taken }\end{array}$ \\
\hline Guideline & Sensitivity & Specificity & $\begin{array}{c}\text { takn } \\
\text { II }\end{array}$ \\
III & $1370(82)$ & $1175(39)$ & $3192(69)$ \\
IV & $1525(92)$ & $590(20)$ & $3932(84)$ \\
\hline & $1497(90)$ & $698(23)$ & $3796(82)$ \\
\hline
\end{tabular}

The performance of a discriminant function may be assessed by comparing the predicted category with the category defined by the $x$ rayo findings. Table I shows the performance of the five discriminant functions applied to the population of 4659 patients. All the discriminant functionso identified correctly only about 500 (about $30 \%$ ) of the 1662 patients with a fracture (that is, they had a sensitivity of about $30 \%$ ) and falsely labelled $\sigma$ about 200 (about 7\%) of the 2997 patients without a bony injury as having a fracture (that is, a specificity of about $93 \%$ ). In other words, if any of these five combinations of symptoms and signs had been used as a guideline $N$ although radiography would have been avoided in about 3950 patients $(85 \% \mathrm{~N}$ of the population), about $1100(66 \%)$ of the 1662 patients with a fracture would not have been identified, and about $200(6.7 \%)$ of the 2997 without bony injury would have been referred for radiography.

Table II summarises the performance of the Boolean protocols. Compared $\frac{\bar{\Phi}}{\mathscr{C}}$ with the discriminant functions, their sensitivities of $80-90 \%$ indicate that they correctly identified a much higher proportion of patients with fractures. Between 160 and 330 of the 1662 fractures would have been missed compared with 1100 using the best discriminant function protocol. As the figures of $20-40 \%$ for specificity show, however, the Boolean protocols $\frac{\mathbb{D}}{\mathbb{D}}$ incorrectly suggested the presence of a fracture in many more of those without bony injury (between $1800-2400$ in the population studied compared with 200 using the discriminant function protocol). This would result in many more patients having radiographs taken, 3260-3960 (70-85\%) compared with $650-745$ (14-16\%).

Results using weighting factors are not presented in the discriminant 
function analysis - that is, the "cost" of missing a case was considered to be the same as the "cost" of performing radiography on a patient unnecessarily - because information on the costs and benefits of false positives and false negatives in the investigation of possible fractures was not available. Guidelines were investigated with adjustments to the prior probabilities of a fracture in such a way that the cost of a false negative was considered to be between two and four times that of a false positive. The resulting guidelines classified almost all the population as having fractures-that is, they had sensitivities of almost $100 \%$ but specificities of almost $0 \%$.

\section{Discussion}

Brand et al produced an algorithm for improving the use of radiography of arms and legs in the United States that showed that the numbers of radiographs of arms and legs could be reduced by $12 \%$ and $19 \%$, respectively. ${ }^{22}$ The authors estimated that the algorithm could reduce the cost of such radiography by between $\$ 79 \mathrm{~m}$ and $\$ 139 \mathrm{~m}$ yearly in the United States. They showed that their guideline performed very well in patients with arm injuries, detecting 109 of the 112 fractures in the study population, although only 280 of 524 patients had radiographs taken. ${ }^{22}$ The fracture yield in their population, however, was only $21 \%$ compared with $36 \%$ in our study. A lower yield generally implies less selective use of an investigation and therefore a greater opportunity for a guideline to make current practice more efficient.

Our results confirm an earlier suggestion that radiography of arms and legs in England and Wales is already being restricted to those patients who are considered to have a definite or suspected fracture on the basis of symptoms and signs. ${ }^{15}$ Further reduction in the use of radiography in accident and emergency departments in England and Wales using guidelines based on the aggregate data from all eight centres would be achieved only at the risk of missing an unacceptably high proportion of fractures.

Using computer simulations of the effects of several algorithms, we investigated whether guidelines for the use of radiography might improve the current selection of patients for radiography. Our findings may be accounted for by the imprecision inherent in the clinical data available to the doctor. This hypothesis suggests that clinical practice, because of its dependence on biological data that are often variable, may not always operate as efficiently as many non-clinical activities in the health service, and that the uncritical pursuit of clinical efficiency should be avoided.

The fracture yield observed in this study suggests that substantial selection is already taking place, and therefore the opportunity for a guideline to improve current practice is correspondingly limited. In this study evaluation showed that a particular clinical practice was probably as good as it could be in view of current knowledge.

In contrast to skull radiography in patients with head injury and routine preoperative chest radiography ${ }^{71-13}$ 23-27 the cost per clinically important abnormality detected by radiography of the arms and legs is very low at about $£ 40 .{ }^{16}$ In addition, the data on results of radiography after reattendance at an accident and emergency department indicate that only one out of a 1000 fractures was undetected at first examination. ${ }^{16}$ Thus it seems that in England and Wales, even in the absence of explicit guidelines, a clinically satisfactory and cost effective selection of those who need radiographs of arms and legs from all those with injured arms and legs is already practised.

The study on which this paper is based was carried out in 1985 under the guidance of the Royal College of Radiologists working party on the effective use of diagnostic radiology, whose members were: Dr M E Abrams, Professor E Rhys Davies, Mr W P Ennis, Professor K T Evans, Dr P M Hacking, Dr A E Hugh, Dr J W Laws, Dr M R McNulty, Professor C J Roberts, and Dr G R Sutherland.

We thank all those connected with the study in the participating centres, particularly Dr L A Wiliams, Dr M Prescott, Dr S Field, Mr E P Abson, Dr J E Williams, Dr A Barker, Dr I P Maddison, Dr S Tachakra, Dr E Roebuck, Dr S H Dave, Dr P M Hacking, Mr A K Maitra, Dr R M Paxton, Mr I P Stewart, Dr B Clay, and Mr M C T Morrison, and the Department of Health and Social Security for financial support.

Details of guidelines referred to in this paper are available from $\operatorname{Dr} M \mathrm{C}$ Charny.

\section{References}

1 Hall FM. Overutilization of radiological examinations. Radiology 1976;120:443-8. 2 Abrams HL. The "overutilization" of $x$-rays. N Engl f Med 1979;300:1213-6.

3 Fineberg HV, Hiatt HH. Evaluation of medical practices: the case for technology assessment. $N$ Engl f Med 1979;301:1086-91.

4 Wrighton RJ, Oliver RM. Trends in radiological practice in the NHS. Health Trends 1980;12: $21-4$.

5 Welsh Office. Health and personal social services statistics for Wales. Cardiff: Welsh Office, 1983.

6 Department of Health and Social Security. Health and personal social services statistics for England 1982. London: HMSO, 1982.

7 Roberts CJ. The effective use of diagnostic radiology. $7 R$ Coll Physicians Lond 1984;18:62-5. 8 Sherwood T. Resources and decisions in clinical radiology. If Epidemiol Community Health 1979;33:59-65

9 Sandler G. Costs of unnecessary tests. Br Med J 1979;ii:21-4.

10 Ashley JSA, Pasker P, Beresford JC. How much clinical investigation? Lancet 1972;i:890-2.

11 Royal College of Radiologists. A study of the utilisation of skull radiography in 9 accident-andemergency units in the UK. Lancet 1980;ii:1234-7.

12 Royal College of Radiologists. Costs and benefits of skull radiography for head injury. Lancet 1981;ii:791-5.

13 Royal College of Radiologists. Patient selection for skull radiography in uncomplicated head injury. Lancet 1983;i:115-8.

4 Royal College of Radiologists. Pre-operative chest radiology. Lancet 1979;ii:82-6.

15 Hayward MWJ, Hayward C, Ennis WP, Roberts CJ. A pilot evaluation of radiography of the acute abdomen. Clin Radiol 1984:35:289-91.

16 Royal College of Radiologists Working Party. Radiography of injured arms and legs in eight accident and emergency units in England and Wales. Br Med $\mathcal{F} 1985$;291:1325-8.

17 Holder AR. Non-negligent failure to take x-ray films. JAMA 1972;219:1259-60.

18 Anonymous. SPSS-X users guide. New York: McGraw-Hill, 1983.

19 Anonymous. SPSS-X statistical algorithms. Chicago: SPSS Inc, 1983.

20 Fleiss JL. Statistical methods for rates and proportions. New York: John Wiley, 1973.

21 Myers A, Brand DA, Dove HG, Dolan TF Jr. A technique for analyzing clinical data to provide patient management guidelines: a study of meningitis in children. Am $\mathcal{J}$ Dis Child 1978;132: 25-9.

22 Brand DA, Frazier WH, Kohlhepp WC, et al. A protocol for selecting patients with injured extremities who need x-rays. N Engl f Med 1982;306:333-9.

23 Roberts CJ, Fowkes FGR, Ennis WP, Mitchell M. Possible impact of audit on chest x-ray requests for surgical wards. Lancet 1983;ii:446-8.

24 Roberts CJ. Towards the effective use of diagnostic radiology in surgical practice: discussion paper. I $R$ Soc Med 1983;76:755-9.

25 Roberts CJ, Fowkes FGR. Introducing guidelines in clinical practice. Journal of Effective Health Care 1984;1:313-21.

26 Roberts CJ. The evaluation of diagnostic services. In: Holland WW, ed. The evaluation of health care. Oxford: Oxford University Press, 1983.

27 World Health Organisation. A rational approach to radiodiagnostic investigations. WHO Tech Rep Ser 1983;689:14.

(Accepted 4 November 1986)

\section{What seating is advised for a patient with chronic back pain and sciatica?}

Soft seats that look deceptively comfortable should be avoided. The seat should be horizontal with firm padding. The height should be sufficient to allow both feet to be placed comfortably on the floor, with knees flexed at right angles. The edge of the seat should extend to a position about 6-8 inches $(15-20 \mathrm{~cm})$ behind the knees to avoid pressure on the calves. The back rest should support the lumbar spine at a distance of about 4-6 inches $(10-15 \mathrm{~cm})$ from the seat and permit a flat lumbar curve. Arm rests at the level of the elbows will add to comfort.-LESLIE KLENERMAN, consultant orthopaedic surgeon, London.

Jayson MIV. Back pain—the facts. Oxford: Oxford University Press, 1981:134.

\section{How common is it for the umbilical cord to coil round the body of a fetus?}

It used to be said that the incidence of this complication was $0.5 \%$, but in the 1960s an American survey of 17190 deliveries found that the cord was coiled round the body in 339 cases-an incidence of about $2 \% .^{1}$ There is an increased risk of abruptio placentae when this happens, ${ }^{1}$ and in one such case maternal death has occurred from amniotic fluid embolism. ${ }^{2}$ Entanglement of the cord may cause fetal death even without placental abruption. ${ }^{3}$ Serious non-fatal sequels may also occur: a recent report has described neonatal brachial artery occlusion due to a cord wound tightly round the baby's arm. ${ }^{4}$ The most common type of cord complication, however, is when the cord is coiled round the baby's neck; this occurs in up to a third of all deliveries and may cause fetal bradycardia and low one minute Apgar scores, though it only rarely causes stillbirth. ${ }^{13}$ All these complications are more common when the cord is abnormally long. ${ }^{13}$-JAMES OWEN DRIFE, senior lecturer in obstetrics and gynaecology, Leicester.

1 Spellacy WN, Gravem H, Fisch RO. The umbilical cord complications of true knots, nuchal coils, and cords around the body. Am J Obstet Gynecol 1966;94:1136-42.

2 Corridan M, Kendall ED, Begg JD. Cord entanglement causing premature placental separation and amniotic fluid embolism: case report. Br f Obstet Gynaecol 1980;87:935-40. 3 Elhassani SB. The umbilical cord: care, anomalies, and diseases. Southern Med $\mathcal{f}$ 1984;77:730-6. 3 Couser RJ, Mammel MC, Coleman M, Boros SJ. Neonatal brachial artery occlusion from an
4 , umbilical cord tourniquet. J Pediatr 1984;104:286-9. 